\title{
Growing Together and Growing Apart: Regional and Sex Differences in the Lifespan Developmental Trajectories of Functional Homotopy
}

\author{
Xi-Nian Zuo, ${ }^{1,2}$ Clare Kelly, ${ }^{1}$ Adriana Di Martino, ${ }^{1,3}$ Maarten Mennes, ${ }^{1}$ Daniel S. Margulies, ${ }^{4}$ Saroja Bangaru, ${ }^{1}$ \\ Rebecca Grzadzinski, ${ }^{1}$ Alan C. Evans, ${ }^{5}$ Yu-Feng Zang, ${ }^{2}$ F. Xavier Castellanos, ${ }^{1,6}$ and Michael P. Milham ${ }^{1,6}$ \\ ${ }^{1}$ Phyllis Green and Randolph Cōwen Institute for Pediatric Neuroscience, New York University Langone Medical Center, New York, New York 10016, ${ }^{2}$ State \\ Key Laboratory of Cognitive Science and Learning, Beijing Normal University, Beijing 100875, China, ${ }^{3}$ Division of Child and Adolescent Neuropsychiatry, \\ Department of Neuroscience, University of Cagliari, 09126 Cagliari, Italy, ${ }^{4}$ Max Planck Institute for Human Cognitive and Brain Sciences, D-04103 Leipzig, \\ Germany, ${ }^{5} \mathrm{McC}$ Connell Brain Imaging Center, Montreal Neurological Institute, McGill University, Montreal, Québec, Canada, H3A 2B4, and ${ }^{\circ}$ athan Kline \\ Institute for Psychiatric Research, Orangeburg, New York 10962
}

Functional homotopy, the high degree of synchrony in spontaneous activity between geometrically corresponding interhemispheric (i.e., homotopic) regions, is a fundamental characteristic of the intrinsic functional architecture of the brain. However, despite its prominence, the lifespan development of the homotopic resting-state functional connectivity (RSFC) of the human brain is rarely directly examined in functional magnetic resonance imaging studies. Here, we systematically investigated age-related changes in homotopic RSFC in 214 healthy individuals ranging in age from 7 to 85 years. We observed marked age-related changes in homotopic RSFC with regionally specific developmental trajectories of varying levels of complexity. Sensorimotor regions tended to show increasing homotopic RSFC, whereas higher-order processing regions showed decreasing connectivity (i.e., increasing segregation) with age. More complex maturational curves were also detected, with regions such as the insula and lingual gyrus exhibiting quadratic trajectories and the superior frontal gyrus and putamen exhibiting cubic trajectories. Sex-related differences in the developmental trajectory of functional homotopy were detected within dorsolateral prefrontal cortex (Brodmann areas 9 and 46) and amygdala. Evidence of robust developmental effects in homotopic RSFC across the lifespan should serve to motivate studies of the physiological mechanisms underlying functional homotopy in neurodegenerative and psychiatric disorders.

\section{Introduction}

Invigorated by the new era of discovery science for human brain function (Van Horn and Gazzaniga, 2002; Biswal et al., 2010), researchers are rediscovering old principles with new enthusiasm. One such principle is brain functional homotopy: the high degree of synchrony in spontaneous activity between geometrically corresponding interhemispheric (i.e., homotopic) regions. Robust homotopic resting-state functional connectivity (RSFC) is a key characteristic of the intrinsic functional architecture of the brain (Salvador et al., 2008; Stark et al., 2008), an unsurprising observation given the high proportion of callosal fibers dedicated to homotopic connectivity (Innocenti, 1986). Despite the prominence of homotopic RSFC, few systematic examinations exist.

\section{Received May 21, 2010; revised Aug. 2, 2010; accepted Aug. 26, 2010}

This research was partially supported by National Institute of Mental Health Grant R01MH083246 and grants from Autism Speaks, the Stavros Niarchos Foundation, the Leon Levy Foundation, and the endowment provided by Phyllis Green and Randolph Cōwen. We thank all participants for their cooperation and Jonathan Adelstein for editorial assistance.

This article is freely available online through the J Neurosci Open Choice option.

Correspondence should be addressed to Xi-Nian Zuo or Michael P. Milham, Phyllis Green and Randolph Cōwen Institute for Pediatric Neuroscience, New York University Langone Medical Center, 215 Lexington Avenue, 14th Floor, New York, NY 10016. E-mail: xinian.zuo@nyumc.org or michael.milham@nyumc.org.

DOI:10.1523/JNEUROSCI.2612-10.2010

Copyright $\odot 2010$ the authors $\quad$ 0270-6474/10/3015034-10\$15.00/0
Although homotopic RSFC is observed ubiquitously in the brain, systematic regional variation exists (Salvador et al., 2008). Consistent with models of hierarchical processing and hemispheric specialization for information processing (Mesulam, 1998), sensorimotor cortex exhibits strong homotopic RSFC, whereas RSFC between homotopic prefrontal and temporoparietal association areas is weaker (Stark et al., 2008). Others have also identified lateralized networks associated with higher cognitive functions (Beckmann et al., 2005; Damoiseaux et al., 2006; Liu et al., 2009; Yan et al., 2009).

RSFC approaches are sensitive to maturational processes throughout the lifespan: several studies have demonstrated developmental shifts from diffuse short-range RSFC in childhood to the increasingly focal, long-range RSFC in young adulthood (Fair et al., 2007, 2008; Fransson et al., 2007; Kelly et al., 2009; Supekar et al., 2009). Subtle changes in RSFC continue throughout adulthood (Biswal et al., 2010), with deterioration becoming evident after age 60 years (Andrews-Hanna et al., 2007). Given the maturational trajectory of the corpus callosum (Paus et al., 1999; Schmithorst and Yuan, 2010), robust age-related changes in homotopic RSFC are likely. This hypothesis was prompted by the strong correspondence (Hagmann et al., 2008), but not 1:1 identity (Vincent et al., 2007; Roy et al., 2009), between structural 
and functional connectivity. Previous studies provide conflicting results: whereas Kelly et al. (2009) observed age-related increases in the lateralization of anterior cingulate networks, Fair et al. (2008) found little evidence of age-related changes in homotopic RSFC among default network regions. Direct investigation of the development of homotopic RSFC is thus warranted.

Here we provide a comprehensive examination of lifespan trajectories of homotopic RSFC using a voxelwise measure called "voxel-mirrored homotopic connectivity" (VMHC). VMHC quantifies the RSFC between each voxel in one hemisphere and its mirrored counterpart in the other. Our sample comprises 214 individuals aged 7-85 years. Following morphometric studies of brain development (Sowell et al., 2003; Toga et al., 2006; Shaw et al., 2008; Giorgio et al., 2010; Lebel et al., 2010; Westlye et al., 2010), we distinguish regions exhibiting cubic, quadratic, and linear developmental trajectories.

We hypothesized that homotopic RSFC would exhibit strong developmental effects with age-related decreases in homotopic RSFC in areas supporting higher-order cognitive processes (Toga and Thompson, 2003). Based on evidence of decreasing lateralization in normal aging (Cabeza et al., 2002; Buckner, 2004; Li et al., 2009; Park and Reuter-Lorenz, 2009), we also predicted quadratic or cubic age-related changes in homotopic RSFC.

\section{Materials and Methods}

\section{Subjects and imaging protocols}

A total of 214 right-handed participants ranging in age from 7 to 85 years (mean age, $27.7 \pm 16.6$ years; 96 males) were scanned at two centers [New York University (NYU) and Montreal Neurological Institute (MNI), the latter as part of International Consortium for Brain Mapping (ICBM)]. The corresponding institutional review boards approved the research protocols. Informed consent was obtained before participation. Participants had no history of neurological and psychiatric disease based on self-report for the adults and on parent and child interviews for the children and adolescents ( $<18$ years).

At NYU, data from 163 typically developing participants ranging in age from 7 to 49 years (mean age, $22.3 \pm 10.7$ years; 74 males) were collected using a Siemens Allegra 3.0 tesla scanner at the NYU Center for Brain Imaging. Two imaging sequences were used to collect the restingstate data: (1) a single-shot echo planar imaging (EPI) sequence [197 time points; repetition time (TR), $2000 \mathrm{~ms}$; echo time (TE), $25 \mathrm{~ms}$; flip angle, $90^{\circ}$; 39 slices; voxel size, $3 \times 3 \times 3 \mathrm{~mm}^{3}$ ] was used for 82 participants; and (2) a multi-echo EPI sequence (180 time points; TR, $2000 \mathrm{~ms}$; flip angle, $90^{\circ}$; 33 slices; voxel size, $3 \times 3 \times 4 \mathrm{~mm}^{3}$ ) was used for 81 participants. A high-resolution T1-weighted image using magnetization prepared gradient echo was also obtained for spatial normalization and group-specific template generation.

At the MNI, 86 resting-state functional magnetic resonance imaging (R-fMRI) datasets were obtained by the ICBM. These data are publicly available as part of the 1000 Functional Connectomes project (www.nitrc. org/projects/fcon_1000) (Biswal et al., 2010). Only the 51 scans with full brain coverage were included in the present analyses. Participants were aged between 19 and 85 years (mean age, $45.1 \pm 20.2$ years; 22 males). Imaging information for the ICBM dataset can be found at http://www.nitrc.org/frs/shownotes.php?release_id=916.

\section{Data preprocessing}

A standard data preprocessing strategy was performed using both FSL (for FMRIB Software Library) (http://www.fmrib.ox.ac.uk/fsl/) and AFNI (for Automated Functional Neuro-Imaging) (http://afni.nimh.nih. gov/afni) software. Scripts containing the processing commands used here have been released as part of the 1000 Functional Connectomes Project (http://www.nitrc.org/projects/fcon_1000) (Biswal et al., 2010). Data preprocessing comprised the following: (1) discarding the first four EPI volumes from each resting-state scan to allow for signal equilibration, (2) slice timing correction, (3) three-dimensional motion correction, (4) time series despiking, (5) spatial smoothing with a $6 \mathrm{~mm}$ full-width half-maximum (FWHM) Gaussian kernel, (6) four-dimensional meanbased intensity normalization, (7) bandpass temporal filtering (0.01-0.1 $\mathrm{Hz}$, (8) removing linear and quadratic trends, (9) estimating a nonlinear transformation from individual functional space to MNI152 space $(2 \times$ $2 \times 2 \mathrm{~mm}^{3}$ resolution), and (10) regressing out nine nuisance signals (global mean, white matter, and CSF signals and six motion parameters). The output of these preprocessing steps was a four-dimensional residual functional volume in native functional space, for each participant.

NYU site R-fMRI sequences 1 and 2 differed in the exact steps involved in the computation of the transformation to MNI152 space. Specifically, for sequence 2 , functional to anatomical coregistration was improved by intermediate registration to a low-resolution EPI image and B0 unwarping.

\section{Voxel-mirrored homotopic connectivity computation}

To account for differences in the geometric configuration of the cerebral hemispheres, we refined the spatial normalization from individual anatomical to MNI152 space. First, all 214 normalized T1 images were averaged to create a mean normalized T1 image. This image was then averaged with its left-right mirrored version to generate a group-specific symmetrical template. Second, we refined the nonlinear registration to standard space for each individual using the symmetrical template. We then applied this refined transformation to the symmetrical brain template to each participant's four-dimensional residual functional data.

Homotopic RSFC was computed as the RSFC between any pair of symmetric interhemispheric voxels. Specifically, in the symmetrical brain space, we calculated the Pearson's correlation coefficient between the residual time series of each voxel and that of its symmetrical interhemispheric counterpart. Correlation values were then Fisher $Z$-transformed. To reduce the potential contributions of white matter, CSF and other non-gray matter locations to homotopic RSFC, Fisher $Z$-values were weighted by the voxelwise gray matter tissue probability, according to the gray matter tissue previously included with FSL. Because this step scales the $Z$-value of each voxel by a single gray matter probability value across all individuals, it has no impact on interindividual (i.e., developmental) effects. The resultant values were referred to as the VMHC and were used for subsequent group-level analyses.

\section{Group-level analyses}

We examined age-related changes in VMHC using general linear model analyses aimed at detecting regional and sex differences in developmental trajectory.

Developmental trajectories. We aimed to detect linear, quadratic, and cubic changes in homotopic RSFC reflecting differences in age, using three multiple linear regressions that modeled mean VMHC and (1) age, (2) age $^{2}$, and (3) age $^{3}$ as predictors, along with six other covariates described in detail below. For example, the model aimed at detecting cubic changes can be formulated as

$$
\begin{aligned}
\mathrm{VMHC}_{i j} & =\text { mean }+\beta_{i 1} \times \text { age }+\beta_{i 2} \times a g e^{2}+\beta_{i 3} \times a g e^{3}+\beta_{i 4} \\
& \times \text { center }+\beta_{i 5} \times \text { sequence }+\beta_{i 6} \times \text { sex }+\beta_{i 7} \times g \mathrm{VMHC} \\
& +\beta_{i 8} \times \text { rms } \mathrm{HM}+\beta_{i 9} \times \text { diffHM }+e_{i j} .
\end{aligned}
$$

In Equation 1, all variables were demeaned except for the mean effect; the subscript $i$ indexes the individual brain spatial locations, $j$ indicates the participant number, and $e_{i j}$ denotes the random error. At each brain location, we performed three multiple regression models (i.e., linear, quadratic, and cubic) and determined the best model among the three based on Akaike's information criterion (AIC) (Akaike, 1974; Hurvich and Tsai, 1989) (for details, see supplemental data, available at www.jneurosci.org as supplemental material). Identification of age peaks or troughs along the developmental trajectory is critical to the study of timing differences in maturation across brain regions. Accordingly, for those regions exhibiting significant quadratic age effects, we examined the ages associated with the lowest or highest VMHC values (i.e., age peaks along the developmental trajectories). Age peaks were computed using the following equation:

$$
\text { peakAGE }=-\beta_{i 1} / 2 \beta_{i 2} \text {. }
$$



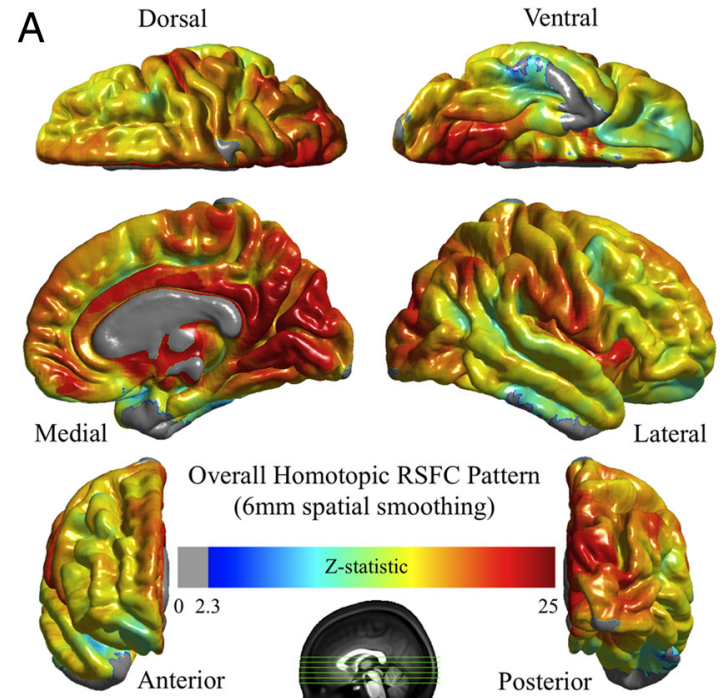
(6mm spatial smoothing)

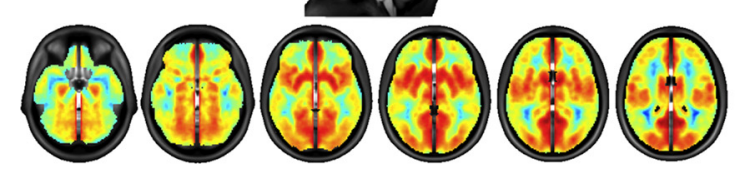

B
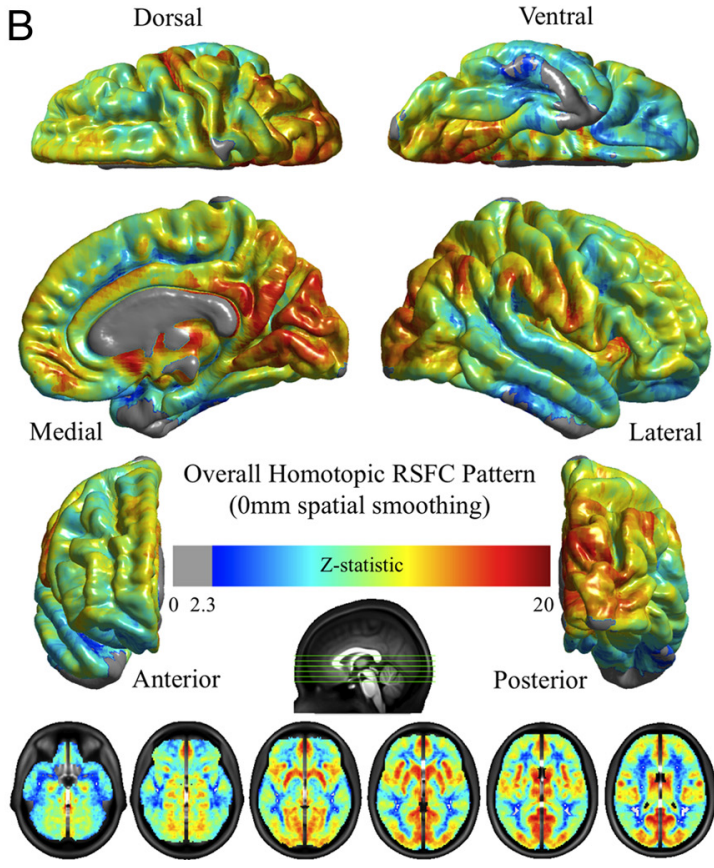

Figure 1. Whole-brain homotopic RSFC pattern. A multiple linear regression models the mean of VMHC at each voxel. One-sample tests on the individual VMHC values were performed. Correction for multiple comparisons was performed within one hemisphere (only one correlation for each pair of homotopic voxels) based on Gaussian random field theory (minimum $Z>2.3$; cluster level, $p<$ 0.05 , corrected). The final statistical maps are visualized as six hemispheric surfaces (cortical regions) and six symmetric axial slices (subcortical regions) for both $6 \mathrm{~mm}$ FWHM ( $\boldsymbol{A}$ ) and $0 \mathrm{~mm}$ FWHM (B) spatial smoothing preprocessing strategies.

Covariates. For each of the models examining the linear and nonlinear effects of age, several additional factors were also modeled: (1) the imaging sequence used (single-shot or multi-echo), (2) the center (NYU or ICBM), (3) sex (male or female), (4) global mean homotopic RSFC (gVMHC) (a continuous variable), and (5) overall head displacement (two continuous variables; see below). Both center and sex factors were modeled as $0-1$ covariates. The sequence factor was modeled as a $0-1-2$ covariate (i.e., 0 , NYU sequence 1 ; 1 , NYU sequence 2 ; 2 , ICBM sequence). Global homotopic RSFC was the mean VMHC across all gray matter voxels with a gray matter mask generated from a gray matter probability image (probability $\geq 40 \%$ ). In considering the potential sensitivity of VMHC measure to head motion, the root mean squares of both overall head displacement (rmsHM) and its temporal derivative (dif$\mathrm{fHM}$ ) were included to adjust for head motion.

Age effects at three spatial scales. For each model, we examined agerelated changes in VMHC at three different spatial levels. At the voxel level, Models 1 were used to test the presence of age effects on VMHC. Multiple comparison corrections were performed within one hemisphere (only one correlation for each pair of homotopic voxels) based on Gaussian random field theory (minimum $Z>2.3$; voxel level, $p<0.01$, uncorrected; cluster level, $p<0.05$, corrected). At the level of global brain or lobar/hierarchical subdivisions, Models 1 did not include gVMHC as a covariate (i.e., reduced models). The mean VMHC across voxels within each large-scale brain unit was extracted for the regression analyses. Bonferroni's corrections were used to correct for multiple comparisons.

Sex differences in VMHC and its development. To identify sex differences in VMHC and sex differences in development of VMHC, we performed another multiple regression analysis:

$$
\begin{aligned}
\mathrm{VMHC}_{i j} & =\text { mean }+\beta_{i 1} \times \text { age }+\beta_{i 2} \times \text { sex }+\beta_{i 3} \times(\text { age } \times \text { sex })+\beta_{i 4} \\
& \times \text { center }+\beta_{i 5} \times \text { sequence }+\beta_{i 6} \times g \mathrm{VMHC}+\beta_{i 7} \\
& \times \text { rms HM }+\beta_{i 8} \times \text { diffHM }+e_{i j} .
\end{aligned}
$$

In Model 3, we added the interaction between age and sex, which allowed us to detect voxels showing developmental differences in VMHC between males and females. Focusing on sex, we examined both positive (male $>$ female) and negative (female $>$ male) contrasts as well as positive and negative interactions with age (i.e., development). Multiple comparisons were corrected within one hemisphere (only one correlation for each pair of homotopic voxels) based on Gaussian random field theory (minimum $Z>2.3$; voxel level, $p<0.01$, uncorrected; cluster level, $p<0.05$, corrected).

\section{Results}

Regional variation in voxel-mirrored homotopic connectivity The examination of VMHC, covarying for age and scan site, demonstrated marked regional differences in homotopic RSFC (Fig. 1A) (axial slices are displayed in supplemental Fig. $\mathrm{S} 1$, available at www.jneurosci.org as supplemental material). Robust homotopic connectivity was observed in visual, motor, and somatosensory areas, as well as subcortical regions (basal ganglia, thalamus, and brainstem). In contrast, the prefrontal cortex exhibited weaker homotopic RSFC, particularly dorsolateral and ventrolateral regions implicated in language, attention, or cognitive control. These findings are consistent with previous work from our laboratory (Stark et al., 2008), which was based on analyses of regions of interest. As demonstrated in Figure $1 A$, the VMHC approach permits a greater appreciation of regional variability in homotopic connectivity within larger gyral structures, such as the precentral, postcentral, inferior frontal, and cingulate gyri, than is obtainable using region-of-interest-based approaches (Fair et al., 2008; Stark et al., 2008). Of note, the overall pattern of findings did not markedly depend on the level of spatial smoothing (0 vs $6 \mathrm{~mm}$ FWHM) (Fig. 1B) (for an axial slice view, see supplemental Fig. S1, available at www.jneurosci.org as supplemental material), although spatial smoothing (6 mm FWHM) clearly improved the correspondence between homotopic regions and resulted in greater VMHC strength across participants. 


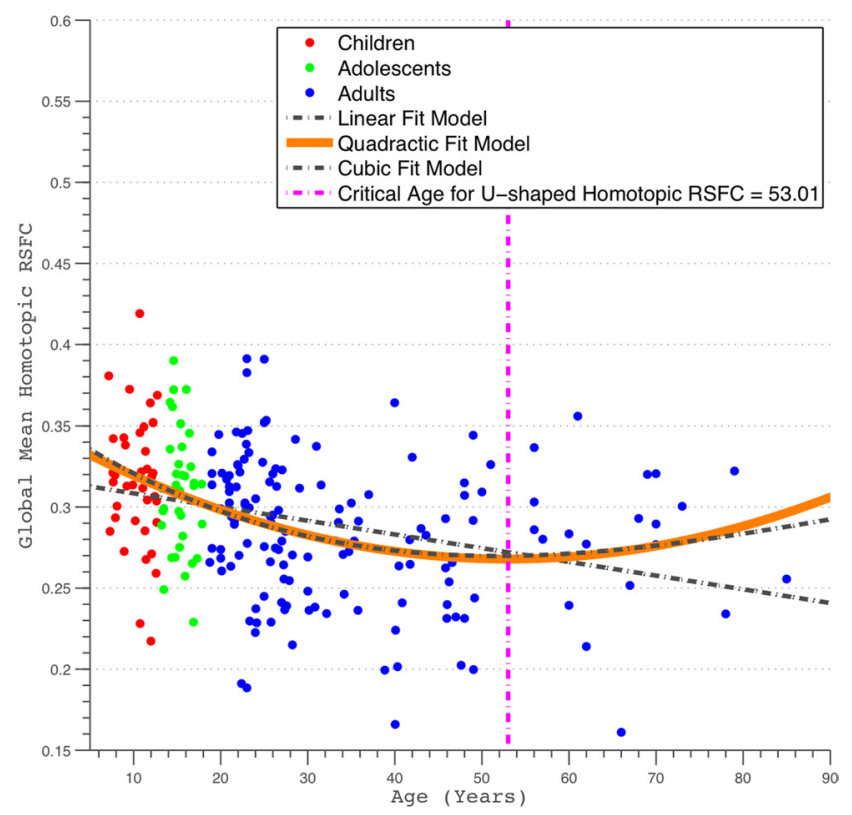

Figure 2. Scatter plots of global homotopic RSFC. The global homotopic RSFC was obtained for each subject by averaging VMHC values across all gray matter voxels within a predefined gray matter mask (40\% threshold) in MNI152 standard space. Linear, quadratic, and cubic curve fittings were applied to these data. Three fit curves are plotted. The quadratic fitting was chosen as the best-fit model using AIC-based model selection, and its peak age is also plotted.

\section{Age-related changes in global homotopic connectivity}

Following developmental studies of human brain morphometry and tractography (Good et al., 2001; Toga et al., 2006; Gong et al., 2009), we examined age-related changes in global homotopic RSFC indexed by mean VMHC (calculated for gray matter only). Global homotopic RSFC decreased with age (Fig. 2) (Pearson's partial correlation, $R^{2}=0.096, r=-0.31, p<2.9 e-6$, $\mathrm{df}=213$ ). Given previous evidence that brain maturation processes follow nonlinear trajectories (Sowell et al., 2003; Toga et al., 2006), we also performed regression analyses that tested for the presence of quadratic and cubic age-related effects. Among these three models (linear, quadratic, and cubic), the best-fitting model by AIC was the quadratic model, suggesting that the relationship between age and VMHC is best described as a nonlinear quadratic. Specifically, global homotopic RSFC decreased over the course of childhood and adolescence but increased again later in life, with an inflection point occurring at age 53 years $\left(R^{2}=0.14, p<\right.$ $9.7 e-8, \mathrm{df}=212$ ). This inflection age is $\sim 24$ years later than that observed for the developmental trajectory of structural homotopy (supplemental Fig. S2, available at www.jneurosci.org as supplemental material) [see voxel-mirrored homotopic morphometry (VMHM) analyses in supplemental data, available at www.jneurosci.org as supplemental material].

\section{Region-specific development of voxelwise homotopic connectivity}

We explored the trajectory of age-related changes in regional homotopic functional connectivity using VMHC. Before testing for the presence of regional differences in the development of VHMC, we removed the contribution of global VMHC at each voxel using linear regression (across participants), thereby ensuring that our findings are truly regionally specific. As for global VHMC, we tested for the presence of linear and quadratic agerelated changes in voxelwise homotopic RSFC. A summary of the developmental trajectories is presented in Figure 3.
Linear regression analyses revealed linear age-related decreases in VMHC for a number of regions, including portions of anterior cingulate gyrus, striatum, parieto-occipital cortex, inferior parietal cortex and parietal operculum, and the ventral posterior precuneus (Fig. 3, green regions) (supplemental Fig. S3A and Table $\mathrm{S} 1$, available at www.jneurosci.org as supplemental material). In contrast, linear age-related increases in VMHC were observed within the central operculum (planum polare), superior frontal sulcus, supplementary motor area, and postcentral gyrus (Fig. 3, pink regions) (supplemental Fig. S3A and Table S1, available at www. jneurosci.org as supplemental material).

Quadratic regression analyses demonstrated nonlinear maturation processes for several regions. For the insula and frontal operculum, fusiform cortex, medial orbital gyrus, inferior frontal sulcus, and parieto-occipital cortex, a positive quadratic (i.e., U-shaped) relationship between age and homotopic RSFC was observed. Age-related decreases in VMHC were observed during childhood and adolescence, whereas increases in VMHC were observed later in life (Fig. 3, red regions) (supplemental Fig. S3B and Table S1, available at www.jneurosci.org as supplemental material). In contrast, for the cerebellum (Crus I), lingual and postcentral gyri, a negative quadratic (i.e., inverted U-shaped) trajectory of homotopic RSFC was observed, with age-related increases in functional connectivity detectable throughout childhood and adolescence but decreased homotopic functional connectivity later in life (Fig. 3, blue regions) (supplemental Fig. S3B and Table S1, available at www.jneurosci.org as supplemental material). The mean inflection ages for voxels exhibiting positive and negative quadratic relationships are 39 and 38 years, respectively (supplemental Fig. S4, available at www.jneurosci.org as supplemental material), although we observed significant variation across voxels (positive range of 13-59, SD of 6; negative range of 18-60, SD of 7).

Cubic analyses revealed voxels within superior frontal cortex (positive effects), putamen, and parahippocampal gyrus (negative effects), showing more complex VMHC maturational patterns. Positive cubic effects indicated that, in the superior frontal cortex, VMHC increased during an initial period from childhood to early adulthood, declined during adulthood, and increased again later in the lifespan (Fig. 3, orange regions) (supplemental Fig. S3C and Table S1, available at www.jneurosci.org as supplemental material). In contrast, negative cubic effects in the putamen and parahippocampal gyrus indicated that VMHC decreased from childhood to early adulthood, increased during adulthood, and then decreased later in the lifespan (Fig. 3, light blue regions) (supplemental Fig. S3C and Table S1, available at www.jneurosci.org as supplemental material).

\section{Lobar and hierarchical characterizations of age-related changes in homotopic connectivity}

To further understand regional differences in the development of homotopic RSFC, we repeated the linear, quadratic, and cubic regression analyses separately for $\mathrm{VMHC}$ measures in each of five brain structural subdivisions and each of six hierarchical subdivisions (Mesulam, 1998). Quadratic fitting was determined as the best model based on AIC model selection criterion. Homotopic RSFC showed significant variation across both lobar and hierarchical structures. Specifically, the temporal lobe and the heteromodal grouping associated with language and attentional control exhibited the lowest homotopic RSFC. In contrast, the occipital lobe and subcortical region exhibited the strongest homotopic functional connectivity.

Brain functional and anatomic subdivisions exhibited different developmental trajectories of homotopic connectivity (Fig. 4). Dif- 
Dorsal
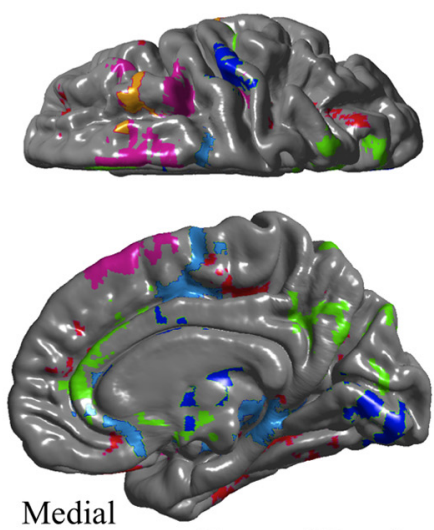

Medial

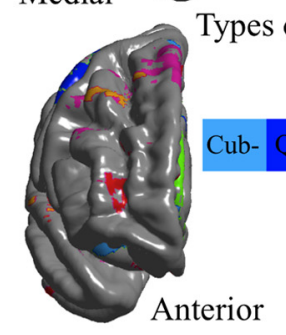
of Homotopic RSFC

- Lin- N.S. Lin+ Qua+ Cub-
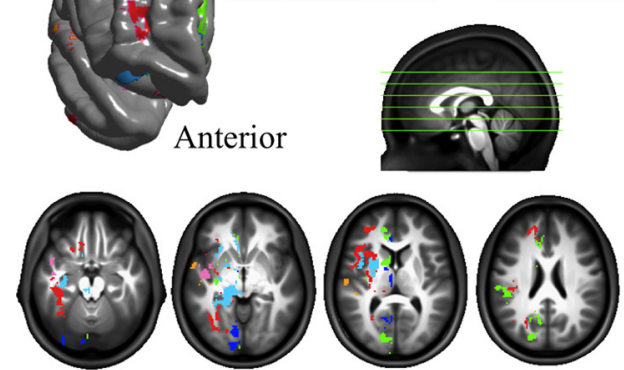

Posterior

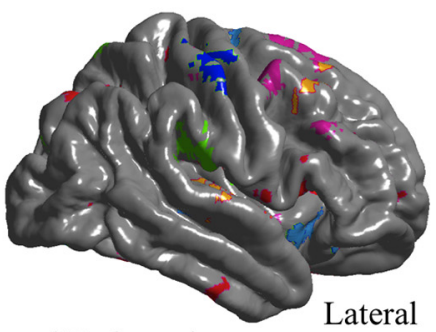

Ventral

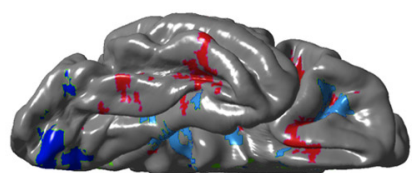

ries
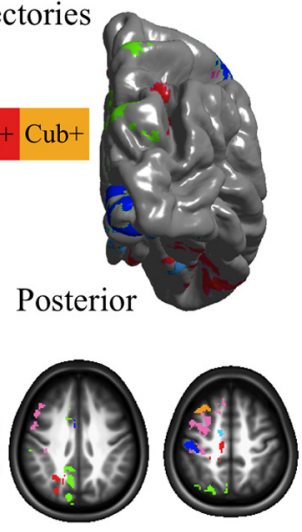
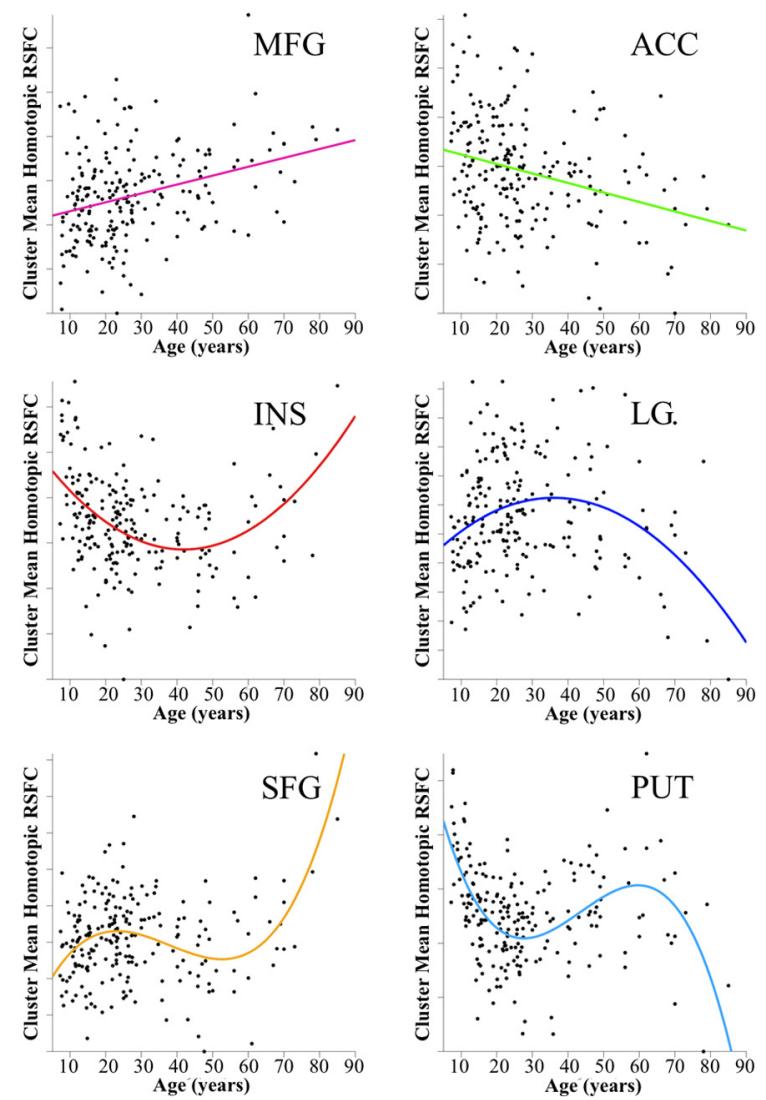

Figure 3. Developmental trajectories of voxelwise homotopic RSFC. Multiple linear regressions model the linear (Lin), quadratic (Qua), and cubic (Cub) age effects on VMHC at each voxel. AIC selected the best model from these three models. One-sample tests on the regression coefficients of age, age $^{2}$, and age ${ }^{3}$ variables were performed for both positive $(+)$ and negative $(-)$ contrasts. Multiple comparisons correction was performed within one hemisphere (only one correlation for each pair of homotopic voxels) based on Gaussian random field theory (minimum $Z>2.3 ;$ cluster level, $p<0.05$, corrected). All six statistical Z-maps ( 3 models $\times 2$ contrasts) were binarized and combined into one summary map to depict different development trajectories of VMHC. The final maps were visualized as six hemispheric surfaces (cortical regions) and six hemispheric axial slices (subcortical regions). The right plots showed the developmental trajectory of a significant cluster for each of six types of trajectories: middle frontal gyrus (MFG: Lin+), anterior cingulate cortex (ACC: Lin-), insula (INS: Qua +), lingual gyrus (LG: Qua-), superior frontal gyrus (SFG: Cub+), and putamen (PUT: Cub-).

ferent inflection ages of homotopic RSFC were observed and followed a high-level to low-level order of development: temporal (47 years), frontal (49 years), parietal (52 years), occipital (64 years), and subcortical (58 years) (Fig. $4 A$ ); heteromodal (49 years), limbic (50 years), unimodal (53 years), paralimbic (55 years), primary (56 years), subcortical (58 years) (Fig. 4B). This ordering is consistent with a "last-in-firstout" aging effect, which has been described previously for measures of white matter integrity (Raz, 2000; Davis et al., 2009). Of note, the maturational patterns of these large brain "structures" strongly resemble the trajectory observed for the global VMHC measure. This was confirmed by the lack of significant linear or quadratic effects of age on the residual homotopic RSFC after global homotopic RSFC was removed by regression from the corresponding mean homotopic RSFC values. However, by decomposing the pattern of global maturation into its regional constituents, the lobar and hierarchical analyses illuminate the regional variations within the overall maturational pattern of homotopic RSFC.
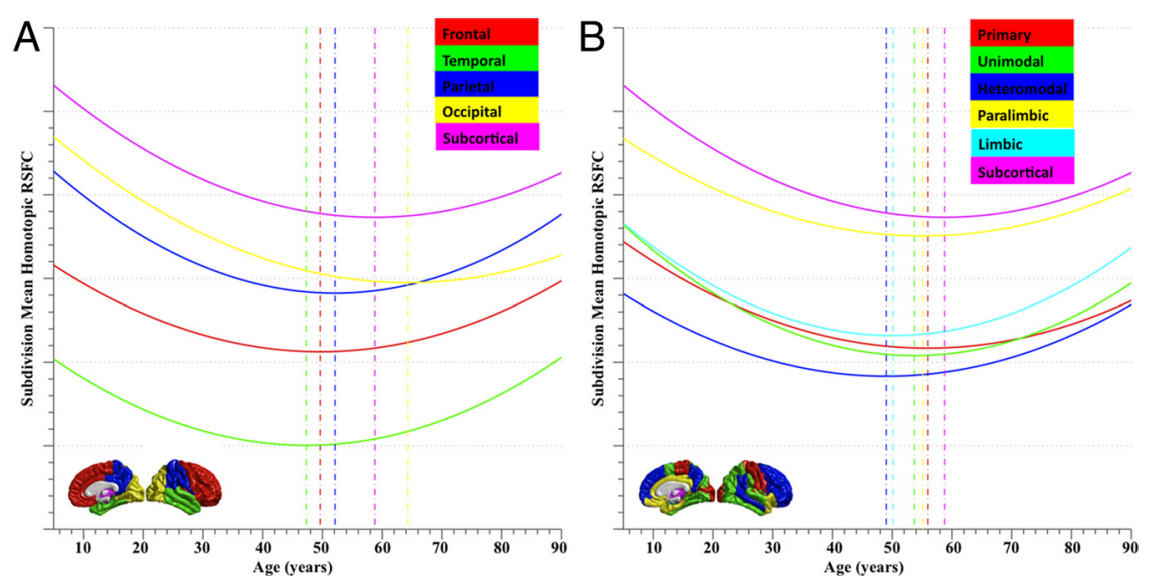

Figure 4. Trajectories of homotopic RSFC in large brain lobar/hierarchical subdivisions. Quadratic regression analyses selected by AIC on age and the mean VMHC of five brain structural subdivisions ( $A$ : frontal, temporal, parietal, and occipital lobes and subcortical region) or six hierarchical subdivisions ( $\boldsymbol{B}$ : primary, unimodal, heteromodal, paralimbic, limbic, and subcortical) were conducted. These large brain units are visualized on the standard brain medial and lateral surfaces. The fit curves and their peak ages are plotted with the same color indicated on the surfaces. All the curve fits are shown significant after Bonferroni's correction.

\section{Sex differences in homotopic connectivity}

Because sex differences in laterality are commonly identified (Cahill, 2006), we examined sex effects on homotopic functional connectivity and its development. Females exhibited stronger homotopic RSFC within posterior cingulate cortex, medial prefron- 

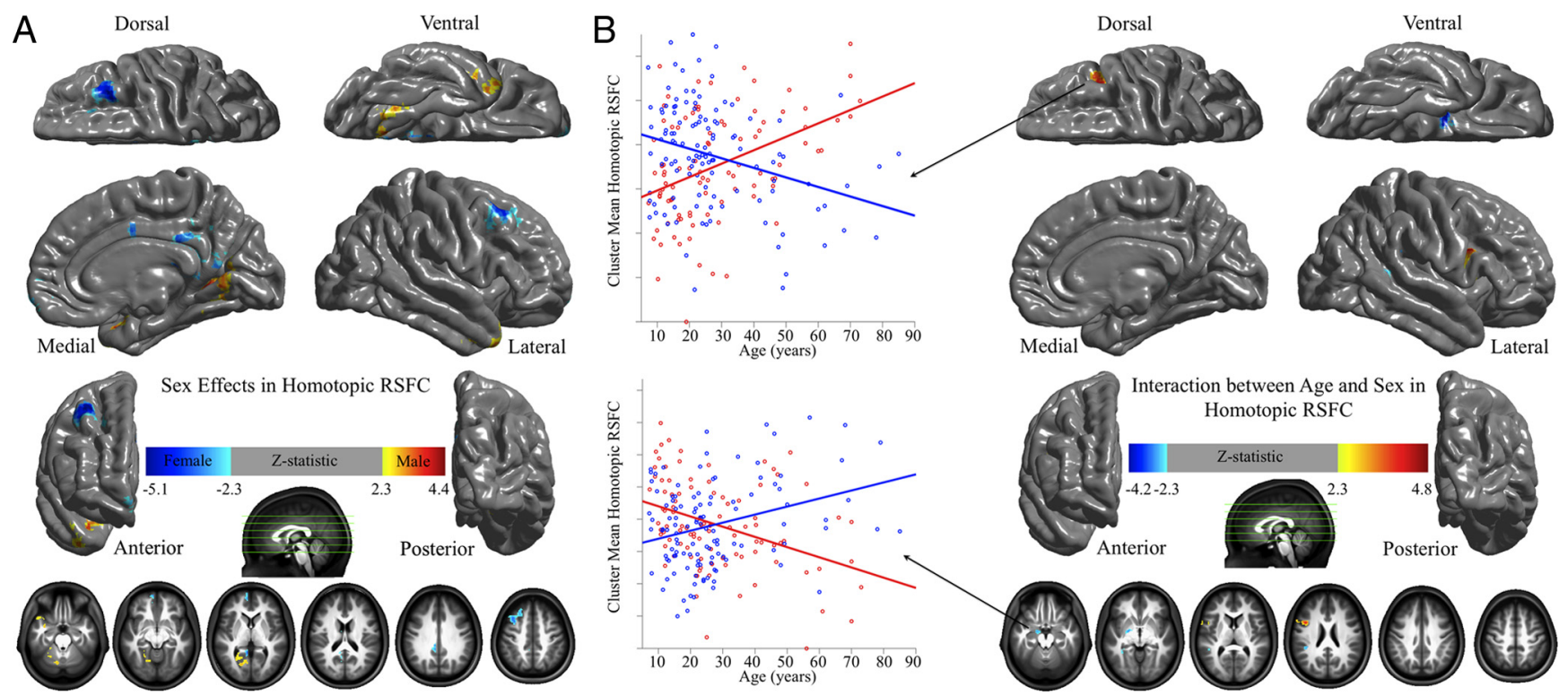

Figure 5. Sex effects of homotopic RSFC. Multiple linear regressions model the age, sex, and their interaction effects on VMHC at each voxel. One-sample tests on regression coefficients of both sex $(\boldsymbol{A})$ and age $\times$ sex interaction $(\boldsymbol{B})$ were performed for both positive and negative contrasts. Multiple comparisons correction was performed within one hemisphere (only one correlation for each pair of homotopic voxels) based on Gaussian random field theory (minimum $Z>2.3$; cluster level, $p<0.05$, corrected). The final statistical maps are visualized as six hemispheric surfaces (cortical regions) and six hemispheric axial slices (subcortical regions). The two scatter plots showed the details of the interactions between age and sex (male, red; female, blue).

tal cortex, and the superior and middle frontal cortex, relative to males. In contrast, males exhibited stronger homotopic RSFC in the cerebellum, parahippocampal gyrus, and fusiform gyrus, relative to females (Fig. 5A). These findings primarily overlapped with the results of our previous examination of sex differences on other R-fMRI measures (Biswal et al., 2010). Interestingly, portions of the dorsolateral prefrontal cortex [Brodmann areas (BA) 9 and 46] exhibited a significant interaction, with age-related increases in VMHC observed for males $(r=0.41, p<0.00004)$, whereas age-related decreases were observed in females $(r=$ $-0.31, p<0.0008)$. In contrast, the amygdala exhibited the opposite pattern of results: age-related decreases in VHMC for males $(r=-0.38, p<0.0002)$ and age-related increases for females $(r=0.30, p<0.001)$ (Fig. 5B). The VMHC scores of the two regions are significantly negatively correlated across individuals $(r=-0.26, p<0.0002)$.

Validation of methods and findings: test-retest reliability, registration, comparability, and structural homotopy

To validate the VMHC method and our findings, we performed several supplementary analyses. Specifically, we examined (1) the test-retest (TRT) reliability of the VMHC measure; (2) the impact of functional-to-structural coregistration on VMHC developmental effects; (3) the impact of combining two datasets; and (4) interhemispheric differences in brain morphometry on our findings. The main findings of these analyses are presented below. We refer the reader to the supplemental data for more details (available at www.jneurosci.org as supplemental material).

First, using our TRT R-fMRI dataset (www.nitrc.org/projects/ nyu_trt) (Shehzad et al., 2009; Zuo et al., 2010a,b), we examined the TRT reliability of VMHC. VMHC showed both short- and long-term fair to excellent $(0.4<$ intraclass correlation $<1.0)$ reliability (Rosner, 2005) across most brain cortical and subcortical regions (Fig. 6).

In a second supplementary analysis, we examined the impact of the functional-to-structural coregistration on VMHC devel- opmental effects. The quality of the functional-to-structural coregistration was significantly correlated with age (full brain, $r=0.2, p<0.003$; midline of brain, $r=0.14, p<0.05$ ) but had only a slight impact on our main findings when it was included as a covariate (supplemental Fig. S5 and other supplemental data, available at www.jneurosci.org as supplemental material).

Third, our comparability analyses suggested the advantages of combining two samples to achieve data spanning the full lifespan and demonstrated the robustness of our results across samples (supplemental Figs. S6, S7; for an additional discussion, see supplemental data, available at www.jneurosci.org as supplemental material). Finally, to examine the extent to which our findings are functionally specific or are driven by interhemispheric differences in gray matter volume, we implemented a voxel-based morphometry-based measure, namely, VMHM and repeated our analyses using VMHM as a voxelwise covariate. The results demonstrate that our findings are not attributable to VMHM, emphasizing their functional specificity (supplemental Fig. S8 and for more details, see supplemental data, available at www.jneurosci. org as supplemental material).

\section{Discussion}

We observed marked and regionally specific age-related changes in functional homotopy. Regions associated with sensory processing and motor control (e.g., secondary auditory, somatosensory, and premotor cortex) showed increased homotopic RSFC with age. In contrast, higher-order cognitive regions (e.g., anterior cingulate, inferior parietal cortex, and precuneus) exhibited decreased homotopic RSFC with age. For several regions, quadratic and cubic age-related effects in interhemispheric RSFC emerged in association with development. Sex-related differences in the developmental trajectories of functional homotopy were detected within dorsolateral prefrontal cortex (BA 9 and BA 46) and amygdala. Overall, our findings demonstrate the potential utility of homotopic RSFC measures for assessing the maturational stage and integrity of human brain function. 

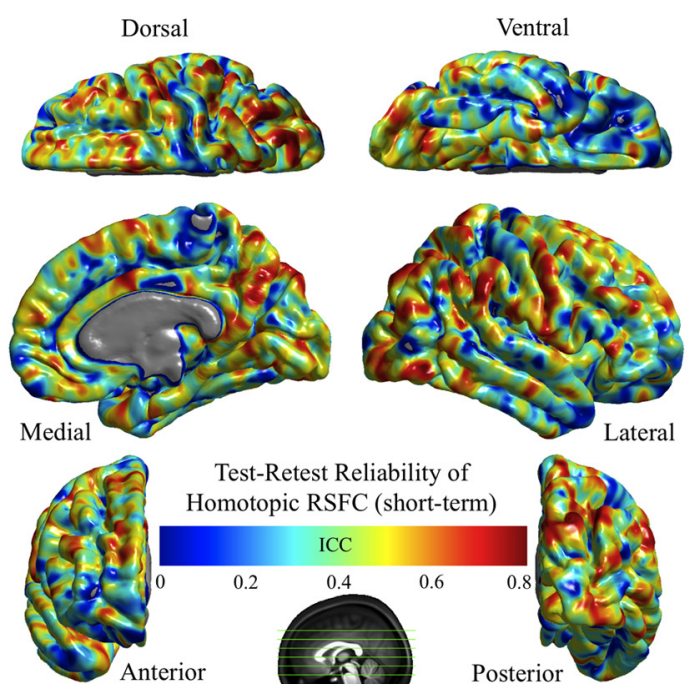

Test-Retest Reliability of omotopic RSFC (short-term)
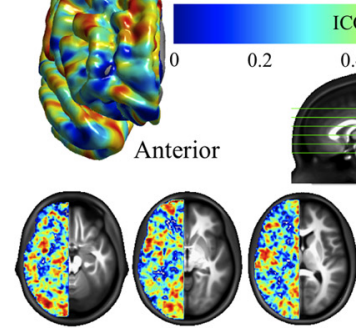
ICC
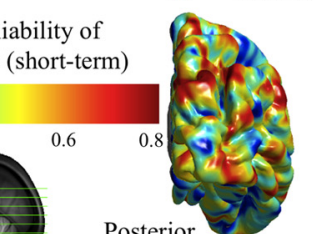
a Posterior

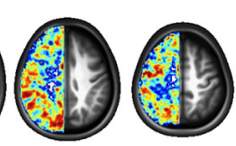

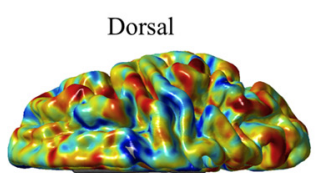
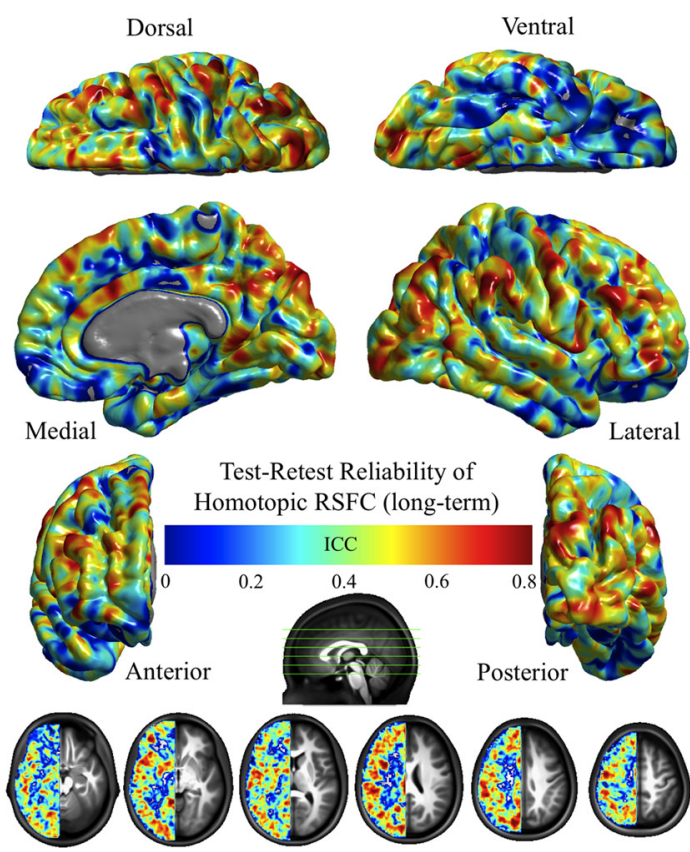

Figure 6. Test-retest reliability of homotopic RSFC. This figure depicts the voxelwise intraclass correlation (ICC) maps showing the intrasession or short-term (A) and intersession or long-term $(\boldsymbol{B})$ test-retest reliability for homotopic RSFC. The final intraclass correlation maps are visualized as six hemispheric surfaces (cortical regions) and six symmetric axial slices (subcortical regions).

\section{Homotopic connectivity: ubiquitous yet regionally specific} Many R-fMRI studies have noted the striking degree of homotopic RSFC (Biswal et al., 1995; Salvador et al., 2008; Stark et al., 2008; Liu et al., 2009; Smith et al., 2009). Because RSFC is thought to reflect both the history and the future of interactions between regions (Fox and Raichle, 2007), we interpret stronger and weaker homotopic RSFC as indexing tendencies toward coordinated or independent processing, respectively. Of course, such correlations do not distinguish excitatory from inhibitory interactions between the hemispheres.

Despite the ubiquity of homotopic RSFC, predictable regional variation in the strength of that connectivity exists. Using a 112-unit brain parcellation, Stark et al. (2008) found that hemispherically specialized frontal and parietal association cortex exhibited weaker homotopic RSFC than sensory or motor regions. Here, we found the strongest homotopic RSFC within motor, premotor, and visual cortex and the weakest in temporal and lateral orbital frontal cortex. We also replicated previous findings of stronger homotopic RSFC in the medial wall relative to the lateral convexity and in posterior relative to anterior regions. The higher resolution afforded by voxelwise analyses in this study revealed subtler distinctions among regions than our previous parcellation unit-based approach (for more extensive discussion, see supplemental texts, available at www. jneurosci.org as supplemental material).

\section{Functional homotopy across the lifespan}

Linear developmental trajectories were observed for the dorsal and perigenual anterior cingulate, ventral precuneus, striatum, posterior parietal, and parieto-occipital cortex. We interpret these decreases as evidence of increasing hemispheric specialization for higher-order cognitive processes, including memory encoding and retrieval (Kail, 1990; Banich and Brown, 2000). In contrast, linear increases in VMHC in the central operculum (planum polare), dorsal and medial premotor areas, and somatosensory cortex may reflect increases in hemispheric cooperation underlying the coordination of complex bimanual function (Donchin et al., 1998; Swinnen, 2002; Marion et al., 2003; Muetzel et al., 2008).
Task-evoked activations often suggest more complex development trajectories. For example, prominent bilateral activations are observable in childhood, decrease in adulthood, and re-emerge in old age (Olesen et al., 2007; Geier et al., 2009; Park and Reuter-Lorenz, 2009; Velanova et al., 2009). We found significant quadratic and cubic effects of age on VMHC in several regions. Portions of medial, lateral and orbital frontal cortex, fusiform gyrus, posterior parietal and intraparietal cortex, and insula showed a quadratic trajectory. These areas subserve diverse higher-order lateralized functions, including language, reading, working memory, and spatial attention. The positive quadratic effect observed in these regions suggests that, although homotopic RSFC decreases with the development of high-level cognition and lateral specialization, this trend reverses after middle age. These findings are consistent with last-in-first-out models (Raz, 2000; Davis et al., 2009), positing the negative impact of advancing age is observed earliest in areas that mature last.

Although task-based studies generally interpret greater bilaterality as reflecting cortical immaturity (Bunge et al., 2002; Wood et al., 2004; Smyser et al., 2010), reasons for the re-emergence of bilaterality in aging are debated. The dedifferentiation hypothesis (Baltes and Lindenberger, 1997) posits that bilateral activation reflects interfering recruitment of contralateral areas, leading to suboptimal task performance. In contrast, compensation hypotheses (Cabeza, 2002; Buckner, 2004; Park and Reuter-Lorenz, 2009) posit that bilateral activation reflects compensatory involvement of the nonpreferred hemisphere. Future developmental studies relating VMHC to cognitive and behavioral performance are needed to adjudicate this debate.

\section{The bases of homotopic functional connectivity and its development}

The age-related trajectories observed for homotopic RSFC resemble those observed in developmental structural studies, particularly of the corpus callosum (Giedd et al., 1999; Thompson et al., 2000; Muetzel et al., 2008), and gray matter asymmetries (Shaw et al., 2009). Developmental changes in the structural ar- 
chitecture of the brain are ascribed to maturational processes, including synaptic pruning, myelination, and learning-dependent reinforcement and synaptic modification (Uhlhaas et al., 2010). Although such processes also likely impact the functional architecture of the brain, caution is required in drawing parallels, because structural and functional connectivity do not correspond in a 1:1 manner (Vincent et al., 2007; Margulies et al., 2009; Roy et al., 2009). In accordance, our supplementary analyses suggested that our findings were not driven by structural homotopy. Robust homotopic RSFC in areas with sparse callosal connectivity, such as primary visual cortex or amygdala, suggests contributions beyond direct callosal connections (Lowe et al., 1998; Cordes et al., 2000; Vincent et al., 2007; Di Martino et al., 2008; Roy et al., 2009). Functional connectivity between these areas may reflect the contribution of subcortical hubs for interhemispheric communication, including the superior colliculi and brainstem (Lyubimov et al., 1983; Savazzi et al., 2007; Uddin et al., 2008). Conversely, the exceptionally strong VMHC observed in subcortical regions may reflect the contributions of bilateral cortical afferent projections rather than direct connectivity (Schulte and Muller-Oehring, 2010). Decreasing RSFC between cortical and subcortical regions over the course of development (Supekar et al., 2009) may thus mediate age-related changes in homotopic RSFC.

\section{Sex matters for homotopic functional connectivity}

We found weaker homotopic RSFC for males in medial and dorsal regions and greater homotopic RSFC in parahippocampal and fusiform gyri. Morphometric studies have detected sex-related differences in the same regions (McDonald et al., 2000; Goldstein et al., 2001; Cahill, 2006), suggesting consistency across structure and function. Interestingly, dorsolateral prefrontal cortex and amygdala exhibited opposite developmental trajectories for males and females. This finding was not driven by structural homotopy, as indicated by our morphometry analyses. Our results suggest sex-related differences in the development of the functional substrates of cognition, emotion, and mental state (Salzman and Fusi, 2010) in regions that differ functionally across the sexes (Zubieta et al., 1999; MacDonald et al., 2000; Cahill et al., 2004).

\section{Implications for neurodegenerative or psychiatric diseases}

Using both R-fMRI and EEG coherence approaches, impaired interhemispheric interaction has been demonstrated in a variety of disorders, including schizophrenia (Spencer et al., 2003; Liang et al., 2006), Alzheimer's disease (Lakmache et al., 1998; Pogarell et al., 2005), multiple sclerosis (Mesulam, 1998; Cover et al., 2006; Lowe et al., 2008), attention deficit hyperactivity disorder (Clarke et al., 2008), and stroke (He et al., 2007; Carter et al., 2010). Altered interhemispheric connectivity between homotopic regions in motor cortex, parietal lobe and insula was also noted in autism (Anderson et al., 2010) as well as disrupted homotopic connectivity of dorsolateral prefrontal cortex in cocaine addiction (C. Kelly, X. N. Zuo, K. Gotimer, C. Cox, L. Lynch, D. Brock, D. Imperati, H. Garavan, J. Rotrosen, F. X. Castellanos, and M. P. Milham, unpublished observations). Building on previous work (Salvador et al., 2008; Stark et al., 2008), our findings suggest that R-fMRI holds significant potential for examinations of atypical interhemispheric interaction, because of both ease of data acquisition and anatomic specificity.

\section{Limitations}

Several limitations should be considered. First, we applied spatial smoothing to improve the spatial correspondence between homotopic regions, which blurs anatomical and functional boundaries (White et al., 2001). Repeating analyses without spatial smoothing reduced VMHC globally, although regional variations were maintained. Thus, the benefits of spatial filtering appear to outweigh the costs.

Second, we pooled R-fMRI datasets from two sites to generate a dataset spanning the majority of the lifespan. The distribution of samples for different age ranges was not equal across the two sites, raising cautions for the interpretation of our findings. One possibility is that overall group differences (i.e., young vs old) confounded our linear model, artifactually suggesting the presence of a linear relationship. However, our comparability analyses, in which we examined age-related effects separately for each dataset, mitigate this concern (for extensive discussions, see supplemental data, available at www.jneurosci.org as supplemental material).

Third, we used cubic, quadratic, and linear fit models to examine age-related changes in VMHC. Parametric curve fitting is sensitive to age-related effects in samples spanning different age ranges (Fjell et al., 2010). Exploration of larger R-fMRI datasets using local curve-fitting approaches may reveal more robust and complex maturational processes (Andersen, 2009; Fjell et al., 2010).

\section{Conclusions}

We demonstrated a robust functional homotopic architecture that exhibits regionally specific age- and sex-related changes across the lifespan. Our analyses captured developmental processes reflected in age-related changes in brain homotopic RSFC that provide strong motivation for future studies aimed at examining the relationship between homotopic RSFC and the corresponding anatomical homotopic "termini." Such work will facilitate discovery of the underlying physiological mechanisms with substantial potential to inform our understanding of development, neurodegenerative conditions, and psychiatric disorders.

\section{References}

Akaike H (1974) A new look at statistical-model identification. IEEE Trans Automat Contr 19:716-723.

Andersen R (2009) Nonparametric methods for modeling nonlinearity in regression analysis. Annu Rev Sociol 35:67-85.

Anderson JS, Druzgal TJ, Froehlich A, DuBray MB, Lange N, Alexander AL, Abildskov T, Nielsen JA, Cariello AN, Cooperrider JR, Bigler ED, Lainhart JE (2010) Decreased interhemispheric functional connectivity in autism. Cereb Cortex. Advance online publication. Retrieved October 12, 2010. doi:10.1093/cercor/bhq190.

Andrews-Hanna JR, Snyder AZ, Vincent JL, Lustig C, Head D, Raichle ME, Buckner RL (2007) Disruption of large-scale brain systems in advanced aging. Neuron 56:924-935.

Baltes PB, Lindenberger U (1997) Emergence of a powerful connection between sensory and cognitive functions across the adult life span: a new window to the study of cognitive aging? Psychol Aging 12:12-21.

Banich MT, Brown WS (2000) A life-span perspective on interaction between the cerebral hemispheres. Dev Neuropsychol 18:1-10.

Beckmann CF, DeLuca M, Devlin JT, Smith SM (2005) Investigations into resting-state connectivity using independent component analysis. Philos Trans R Soc Lond B Biol Sci 360:1001-1013.

Biswal B, Yetkin FZ, Haughton VM, Hyde JS (1995) Functional connectivity in the motor cortex of resting human brain using echo-planar MRI. Magn Reson Med 34:537-541.

Biswal BB, Mennes M, Zuo XN, Gohel S, Kelly C, Smith SM, Beckmann CF, Adelstein JS, Buckner RL, Colcombe S, Dogonowski AM, Ernst M, Fair D, 
Hampson M, Hoptman MJ, Hyde JS, Kiviniemi VJ, Kötter R, Li SJ, Lin CP, et al. (2010) Toward discovery science of human brain function. Proc Natl Acad Sci U S A 107:4734-4739.

Buckner RL (2004) Memory and executive function in aging and AD: multiple factors that cause decline and reserve factors that compensate. Neuron 44:195-208.

Bunge SA, Dudukovic NM, Thomason ME, Vaidya CJ, Gabrieli JD (2002) Immature frontal lobe contributions to cognitive control in children: evidence from fMRI. Neuron 33:301-311.

Cabeza R (2002) Hemispheric asymmetry reduction in older adults: the HAROLD model. Psychol Aging 17:85-100.

Cabeza R, Anderson ND, Locantore JK, McIntosh AR (2002) Aging gracefully: compensatory brain activity in high-performing older adults. Neuroimage 17:1394-1402.

Cahill L (2006) Why sex matters for neuroscience. Nat Rev Neurosci 7:477-484.

Cahill L, Uncapher M, Kilpatrick L, Alkire MT, Turner J (2004) Sex-related hemispheric lateralization of amygdala function in emotionally influenced memory: an FMRI investigation. Learn Mem 11:261-266.

Carter AR, Astafiev SV, Lang CE, Connor LT, Rengachary J, Strube MJ, Pope DL, Shulman GL, Corbetta M (2010) Resting interhemispheric functional magnetic resonance imaging connectivity predicts performance after stroke. Ann Neurol 67:365-375.

Clarke AR, Barry RJ, Heaven PC, McCarthy R, Selikowitz M, Byrne MK (2008) EEG coherence in adults with attention-deficit/hyperactivity disorder. Int J Psychophysiol 67:35-40.

Cordes D, Haughton VM, Arfanakis K, Wendt GJ, Turski PA, Moritz CH, Quigley MA, Meyerand ME (2000) Mapping functionally related regions of brain with functional connectivity MR imaging. AJNR Am J Neuroradiol 21:1636-1644.

Cover KS, Vrenken H, Geurts JJ, van Oosten BW, Jelles B, Polman CH, Stam CJ, van Dijk BW (2006) Multiple sclerosis patients show a highly significant decrease in alpha band interhemispheric synchronization measured using MEG. Neuroimage 29:783-788.

Damoiseaux JS, Rombouts SA, Barkhof F, Scheltens P, Stam CJ, Smith SM, Beckmann CF (2006) Consistent resting-state networks across healthy subjects. Proc Natl Acad Sci U S A 103:13848-13853.

Davis SW, Dennis NA, Buchler NG, White LE, Madden DJ, Cabeza R (2009) Assessing the effects of age on long white matter tracts using diffusion tensor tractography. Neuroimage 46:530-541.

Di Martino A, Scheres A, Margulies DS, Kelly AM, Uddin LQ, Shehzad Z, Biswal B, Walters JR, Castellanos FX, Milham MP (2008) Functional connectivity of human striatum: a resting state FMRI study. Cereb Cortex 18:2735-2747.

Donchin O, Gribova A, Steinberg O, Bergman H, Vaadia E (1998) Primary motor cortex is involved in bimanual coordination. Nature 395:274-278.

Fair DA, Dosenbach NU, Church JA, Cohen AL, Brahmbhatt S, Miezin FM, Barch DM, Raichle ME, Petersen SE, Schlaggar BL (2007) Development of distinct control networks through segregation and integration. Proc Natl Acad Sci U S A 104:13507-13512.

Fair DA, Cohen AL, Dosenbach NU, Church JA, Miezin FM, Barch DM, Raichle ME, Petersen SE, Schlaggar BL (2008) The maturing architecture of the brain's default network. Proc Natl Acad Sci US A 105:4028-4032.

Fjell AM, Walhovd KB, Westlye LT, Ostby Y, Tamnes CK, Jernigan TL, Gamst A, Dale AM (2010) When does brain aging accelerate? Dangers of quadratic fits in cross-sectional studies. Neuroimage 50:1376-1383.

Fox MD, Raichle ME (2007) Spontaneous fluctuations in brain activity observed with functional magnetic resonance imaging. Nat Rev Neurosci 8:700-711.

Fransson P, Skiöld B, Horsch S, Nordell A, Blennow M, Lagercrantz H, Aden U (2007) Resting-state networks in the infant brain. Proc Natl Acad Sci U S A 104:15531-15536.

Geier CF, Garver K, Terwilliger R, Luna B (2009) Development of working memory maintenance. J Neurophysiol 101:84-99.

Giedd JN, Blumenthal J, Jeffries NO, Rajapakse JC, Vaituzis AC, Liu H, Berry YC, Tobin M, Nelson J, Castellanos FX (1999) Development of the human corpus callosum during childhood and adolescence: a longitudinal MRI study. Prog Neuropsychopharmacol Biol Psychiatry 23:571-588.

Giorgio A, Watkins KE, Chadwick M, James S, Winmill L, Douaud G, De Stefano N, Matthews PM, Smith SM, Johansen-Berg H, James AC (2010)
Longitudinal changes in grey and white matter during adolescence. Neuroimage 49:94-103.

Goldstein JM, Seidman LJ, Horton NJ, Makris N, Kennedy DN, Caviness VS Jr, Faraone SV, Tsuang MT (2001) Normal sexual dimorphism of the adult human brain assessed by in vivo magnetic resonance imaging. Cereb Cortex 11:490-497.

Gong G, Rosa-Neto P, Carbonell F, Chen ZJ, He Y, Evans AC (2009) Ageand gender-related differences in the cortical anatomical network. J Neurosci 29:15684-15693.

Good CD, Johnsrude IS, Ashburner J, Henson RN, Friston KJ, Frackowiak RS (2001) A voxel-based morphometric study of ageing in 465 normal adult human brains. Neuroimage 14:21-36.

Hagmann P, Cammoun L, Gigandet X, Meuli R, Honey CJ, Wedeen VJ, Sporns O (2008) Mapping the structural core of human cerebral cortex. PLoS Biol 6:e159.

He BJ, Snyder AZ, Vincent JL, Epstein A, Shulman GL, Corbetta M (2007) Breakdown of functional connectivity in frontoparietal networks underlies behavioral deficits in spatial neglect. Neuron 53:905-918.

Hurvich CM, Tsai CL (1989) Regression and time-series model selection in small samples. Biometrika 76:297-307.

Innocenti G (1986) General organization of callosal connections in the cerebral cortex. In: Cerebral cortex, pp 291-353. New York: Plenum.

Kail R (1990) The development of memory in children. New York: Freeman.

Kelly AM, Di Martino A, Uddin LQ, Shehzad Z, Gee DG, Reiss PT, Margulies DS, Castellanos FX, Milham MP (2009) Development of anterior cingulate functional connectivity from late childhood to early adulthood. Cereb Cortex 19:640-657.

Lakmache Y, Lassonde M, Gauthier S, Frigon JY, Lepore F (1998) Interhemispheric disconnection syndrome in Alzheimer's disease. Proc Natl Acad Sci U S A 95:9042-9046.

Lebel C, Caverhill-Godkewitsch S, Beaulieu C (2010) Age-related regional variations of the corpus callosum identified by diffusion tensor tractography. Neuroimage 52:20-31.

Li Z, Moore AB, Tyner C, Hu X (2009) Asymmetric connectivity reduction and its relationship to "HAROLD" in aging brain. Brain Res 1295: $149-158$.

Liang M, Zhou Y, Jiang T, Liu Z, Tian L, Liu H, Hao Y (2006) Widespread functional disconnectivity in schizophrenia with resting-state functional magnetic resonance imaging. Neuroreport 17:209-213.

Liu H, Stufflebeam SM, Sepulcre J, Hedden T, Buckner RL (2009) Evidence from intrinsic activity that asymmetry of the human brain is controlled by multiple factors. Proc Natl Acad Sci U S A 106:20499-20503.

Lowe MJ, Mock BJ, Sorenson JA (1998) Functional connectivity in single and multislice echoplanar imaging using resting-state fluctuations. Neuroimage 7:119-132.

Lowe MJ, Beall EB, Sakaie KE, Koenig KA, Stone L, Marrie RA, Phillips MD (2008) Resting state sensorimotor functional connectivity in multiple sclerosis inversely correlates with transcallosal motor pathway transverse diffusivity. Hum Brain Mapp 29:818-827.

Lyubimov NN, Baziyan BK, Bochorishvili VN (1983) Role of the midbrain in interhemispheric integration. Neurosci Behav Physiol 13:101-107.

MacDonald AW 3rd, Cohen JD, Stenger VA, Carter CS (2000) Dissociating the role of the dorsolateral prefrontal and anterior cingulate cortex in cognitive control. Science 288:1835-1838.

Margulies DS, Vincent JL, Kelly C, Lohmann G, Uddin LQ, Biswal BB, Villringer A, Castellanos FX, Milham MP, Petrides M (2009) Precuneus shares intrinsic functional architecture in humans and monkeys. Proc Natl Acad Sci U S A 106:20069-20074.

Marion SD, Kilian SC, Naramor TL, Brown WS (2003) Normal development of bimanual coordination: visuomotor and interhemispheric contributions. Dev Neuropsychol 23:399-421.

McDonald B, Highley JR, Walker MA, Herron BM, Cooper SJ, Esiri MM, Crow TJ (2000) Anomalous asymmetry of fusiform and parahippocampal gyrus gray matter in schizophrenia: a postmortem study. Am J Psychiatry 157:40-47.

Mesulam MM (1998) From sensation to cognition. Brain 121:1013-1052.

Muetzel RL, Collins PF, Mueller BA, M Schissel A, Lim KO, Luciana M (2008) The development of corpus callosum microstructure and associations with bimanual task performance in healthy adolescents. Neuroimage 39:1918-1925.

Olesen PJ, Macoveanu J, Tegnér J, Klingberg T (2007) Brain activity related 
to working memory and distraction in children and adults. Cereb Cortex 17:1047-1054.

Park DC, Reuter-Lorenz P (2009) The adaptive brain: aging and neurocognitive scaffolding. Annu Rev Psychol 60:173-196.

Paus T, Zijdenbos A, Worsley K, Collins DL, Blumenthal J, Giedd JN, Rapoport JL, Evans AC (1999) Structural maturation of neural pathways in children and adolescents: in vivo study. Science 283:1908-1911.

Pogarell O, Teipel SJ, Juckel G, Gootjes L, Möller T, Bürger K, Leinsinger G, Möller HJ, Hegerl U, Hampel H (2005) EEG coherence reflects regional corpus callosum area in Alzheimer's disease. J Neurol Neurosurg Psychiatry 76:109-111.

Raz N (2000) Aging of the brain and its impaction on cognitive perfomance: integration of structural and functional findings. In: The handbook of aging and cognition (Craik FIM, Salthouse TA, eds). London: Erlbaum.

Rosner B (2005) Fundamentals of biostatistics, Ed 6. Belmont, CA: Duxbury.

Roy AK, Shehzad Z, Margulies DS, Kelly AM, Uddin LQ, Gotimer K, Biswal BB, Castellanos FX, Milham MP (2009) Functional connectivity of the human amygdala using resting state fMRI. Neuroimage 45:614-626.

Salvador R, Martínez A, Pomarol-Clotet E, Gomar J, Vila F, Sarró S, Capdevila A, Bullmore E (2008) A simple view of the brain through a frequencyspecific functional connectivity measure. Neuroimage 39:279-289.

Salzman CD, Fusi S (2010) Emotion, cognition, and mental state representation in amygdala and prefrontal cortex. Annu Rev Neurosci 33:173-202.

Savazzi S, Fabri M, Rubboli G, Paggi A, Tassinari CA, Marzi CA (2007) Interhemispheric transfer following callosotomy in humans: role of the superior colliculus. Neuropsychologia 45:2417-2427.

Schmithorst VJ, Yuan W (2010) White matter development during adolescence as shown by diffusion MRI. Brain Cogn 72:16-25.

Schulte T, Müller-Oehring EM (2010) Contribution of callosal connections to the interhemispheric integration of visuomotor and cognitive processes. Neuropsychol Rev 20:174-190.

Shaw P, Kabani NJ, Lerch JP, Eckstrand K, Lenroot R, Gogtay N, Greenstein D, Clasen L, Evans A, Rapoport JL, Giedd JN, Wise SP (2008) Neurodevelopmental trajectories of the human cerebral cortex. J Neurosci 28:3586-3594.

Shaw P, Lalonde F, Lepage C, Rabin C, Eckstrand K, Sharp W, Greenstein D, Evans A, Giedd JN, Rapoport J (2009) Development of cortical asymmetry in typically developing children and its disruption in attentiondeficit/hyperactivity disorder. Arch Gen Psychiatry 66:888-896.

Shehzad Z, Kelly AM, Reiss PT, Gee DG, Gotimer K, Uddin LQ, Lee SH, Margulies DS, Roy AK, Biswal BB, Petkova E, Castellanos FX, Milham MP (2009) The resting brain: unconstrained yet reliable. Cereb Cortex 19:2209-2229.

Smith SM, Fox PT, Miller KL, Glahn DC, Fox PM, Mackay CE, Filippini N, Watkins KE, Toro R, Laird AR, Beckmann CF (2009) Correspondence of the brain's functional architecture during activation and rest. Proc Natl Acad Sci U S A 106:13040-13045.

Smyser CD, Inder TE, Shimony JS, Hill JE, Degnan AJ, Snyder AZ, Neil JJ (2010) Longitudinal analysis of neural network development in preterm infants. Cereb Cortex. Advance online publication. Retrieved September 14, 2010. doi:10.1093/cercor/bhq035.

Sowell ER, Peterson BS, Thompson PM, Welcome SE, Henkenius AL, Toga AW (2003) Mapping cortical change across the human life span. Nat Neurosci 6:309-315.

Spencer KM, Nestor PG, Niznikiewicz MA, Salisbury DF, Shenton ME, Mc-
Carley RW (2003) Abnormal neural synchrony in schizophrenia. J Neurosci 23:7407-7411.

Stark DE, Margulies DS, Shehzad ZE, Reiss P, Kelly AM, Uddin LQ, Gee DG, Roy AK, Banich MT, Castellanos FX, Milham MP (2008) Regional variation in interhemispheric coordination of intrinsic hemodynamic fluctuations. J Neurosci 28:13754-13764.

Supekar K, Musen M, Menon V (2009) Development of large-scale functional brain networks in children. PLoS Biol 7:e1000157.

Swinnen SP (2002) Intermanual coordination: from behavioural principles to neural-network interactions. Nat Rev Neurosci 3:348-359.

Thompson PM, Giedd JN, Woods RP, MacDonald D, Evans AC, Toga AW (2000) Growth patterns in the developing brain detected by using continuum mechanical tensor maps. Nature 404:190-193.

Toga AW, Thompson PM (2003) Mapping brain asymmetry. Nat Rev Neurosci 4:37-48.

Toga AW, Thompson PM, Sowell ER (2006) Mapping brain maturation. Trends Neurosci 29:148-159.

Uddin LQ, Mooshagian E, Zaidel E, Scheres A, Margulies DS, Kelly AM, Shehzad Z, Adelstein JS, Castellanos FX, Biswal BB, Milham MP (2008) Residual functional connectivity in the split-brain revealed with restingstate functional MRI. Neuroreport 19:703-709.

Uhlhaas PJ, Roux F, Rodriguez E, Rotarska-Jagiela A, Singer W (2010) Neural synchrony and the development of cortical networks. Trends Cogn Sci 14:72-80.

Van Horn JD, Gazzaniga MS (2002) Opinion: Databasing fMRI studies towards a "discovery science" of brain function. Nat Rev Neurosci 3:314-318.

Velanova K, Wheeler ME, Luna B (2009) The maturation of task set-related activation supports late developmental improvements in inhibitory control. J Neurosci 29:12558-12567.

Vincent JL, Patel GH, Fox MD, Snyder AZ, Baker JT, Van Essen DC, Zempel JM, Snyder LH, Corbetta M, Raichle ME (2007) Intrinsic functional architecture in the anaesthetized monkey brain. Nature 447:83-86.

Westlye LT, Walhovd KB, Dale AM, Bjornerud A, Due-Tonnessen P, Engvig A, Grydeland H, Tamnes CK, Ostby Y, Fjell AM (2010) Differentiating maturational and aging-related changes of the cerebral cortex by use of thickness and signal intensity. Neuroimage 52:172-185.

White T, O'Leary D, Magnotta V, Arndt S, Flaum M, Andreasen NC (2001) Anatomic and functional variability: the effects of filter size in group fMRI data analysis. Neuroimage 13:577-588.

Wood AG, Harvey AS, Wellard RM, Abbott DF, Anderson V, Kean M, Saling MM, Jackson GD (2004) Language cortex activation in normal children. Neurology 63:1035-1044.

Yan H, Zuo XN, Wang D, Wang J, Zhu C, Milham MP, Zhang D, Zang Y (2009) Hemispheric asymmetry in cognitive division of anterior cingulate cortex: a resting-state functional connectivity study. Neuroimage 47:1579-1589.

Zubieta JK, Dannals RF, Frost JJ (1999) Gender and age influences on human brain mu-opioid receptor binding measured by PET. Am J Psychiatry $156: 842-848$.

Zuo XN, Di Martino A, Kelly C, Shehzad ZE, Gee DG, Klein DF, Castellanos FX, Biswal BB, Milham MP (2010a) The oscillating brain: complex and reliable. Neuroimage 49:1432-1445.

Zuo XN, Kelly C, Adelstein JS, Klein DF, Castellanos FX, Milham MP (2010b) Reliable intrinsic connectivity networks: test-retest evaluation using ICA and dual regression approach. Neuroimage 49:2163-2177. 